\title{
Métrologie des propriétés optiques de matériaux massifs : absorption résiduelle, coefficients thermo-optiques, piézo-électriques et électro-optiques.
}

\author{
Jacques Mangin \\ Laboratoire de Physique, Université de Bourgogne, \\ B.P. 47870, F-21078 DIJON CEDEX, FRANCE
}

\begin{abstract}
Résumé. Cet article présente quelques méthodes expérimentales mises en œuvre pour déterminer de la façon la plus précise possible quelques propriétés importantes des matériaux optiques massifs. Les grandeurs examinées ici sont l'absorption résiduelle, les coefficients thermo-optiques, électro-optiques et piézo-électriques. L'accent est mis en particulier sur des techniques de type photo-acoustique pour l'obtention du premier paramètre et sur le développement de techniques interférométriques spécifiques pour la mesure des trois autres coefficients.
\end{abstract}

Mots-clés. Calorimetry, interferometry, piezo-, thermo- and electro-optical effects.

\section{OBJECTIF}

Soumis à une puissance de rayonnement donnée, tout élément optique utilisé dans sa fenêtre de transparence spectrale subit une modification de température dont la valeur dépend de son coefficient d'absorption résiduelle à la longueur d'onde employée. À faible puissance et pour des coefficients petits, qu'on prendra de l'ordre de $0,01 \mathrm{~cm}^{-1}$ pour fixer les idées, cet effet est négligeable et peut être ignoré. Par contre pour des densités de puissance importantes, ce qui est souvent le cas dans les systèmes lasers, l'augmentation de température peut être suffisamment significative pour conduire à une modification conséquente des indices de réfraction $n_{i}$ du matériau, que l'on quantifie par les coefficients thermo-optiques $\mathrm{d} n_{\mathrm{i}} / \mathrm{d} T$. On comprend alors la nécessité d'une détermination très précise de ces paramètres dans la mesure où ils sont nécessairement à prendre en compte pour optimiser au mieux un système optique donné. L'objectif des parties deux et trois de ce travail est de décrire les méthodes expérimentales appropriées pour y parvenir.

Les parties quatre et cinq concernent respectivement la mesure des coefficients piézoélectriques et électro-optiques. On soulignera l'utilisation avantageuse, si possible, de la méthodologie d'étude par interférométrie développée pour la détermination des divers coefficients. Le dilatomètre interférométrique absolu mis au point à cette fin est décrit en partie six et constitue une nouveauté technique, de même que la technique de Fabry-Pérot à balayage thermique pour la détermination de coefficients électro-optiques et la bolométrie piézo-électrique pour celle de faibles absorptions optiques.

Les connaissances pré-requises concernent principalement l'interférométrie optique et les propriétés diélectriques des cristaux.

\section{ABSORPTION RÉSIDUELLE}

L'objectif est de pouvoir déterminer de façon précise des coefficients d'absorption de l'ordre de $1 \% \mathrm{~cm}^{-1}$, ce qui est difficilement accessible par spectrométrie classique. Les méthodes mises en œuvre sont alors de type calorimétrique, usuelles, ou de type photo-acoustique. Ces dernières ne peuvent être employées que pour certaines classes de symétrie s'il s'agit de matériaux cristallins. 


\subsection{Méthodes calorimétriques}

Le principe des techniques calorimétriques consiste à analyser la réponse thermique du matériau soumis à un échelon de puissance de rayonnement dans un intervalle de temps fixé.

\subsubsection{Montage expérimental}

La figure 1 donne le schéma de principe d'un banc couramment utilisé pour mesurer l'absorption d'un matériau à la longueur d'onde émise par un laser.

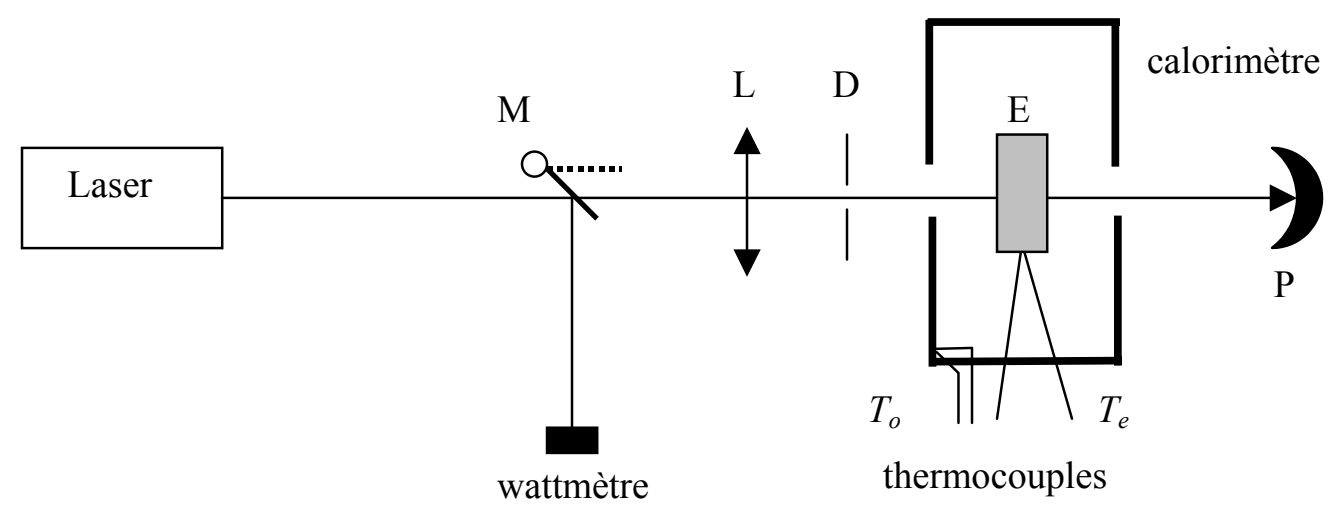

FIG. 1. Schéma de principe de la méthode calorimétrique. L'échantillon E est placé dans un calorimètre et soumis au rayonnement d'un laser dont la puissance est mesurée par un wattmètre, en interposant sur le trajet du faisceau le miroir escamotable M. L représente une lentille de focalisation, D un diaphragme et $\mathrm{P}$ un puits de lumière. $T_{o}$ et $T_{\mathrm{e}}$ sont respectivement les températures de l'enceinte et de l'échantillon, repérées par deux thermocouples.

\subsubsection{Réponse enregistrée}

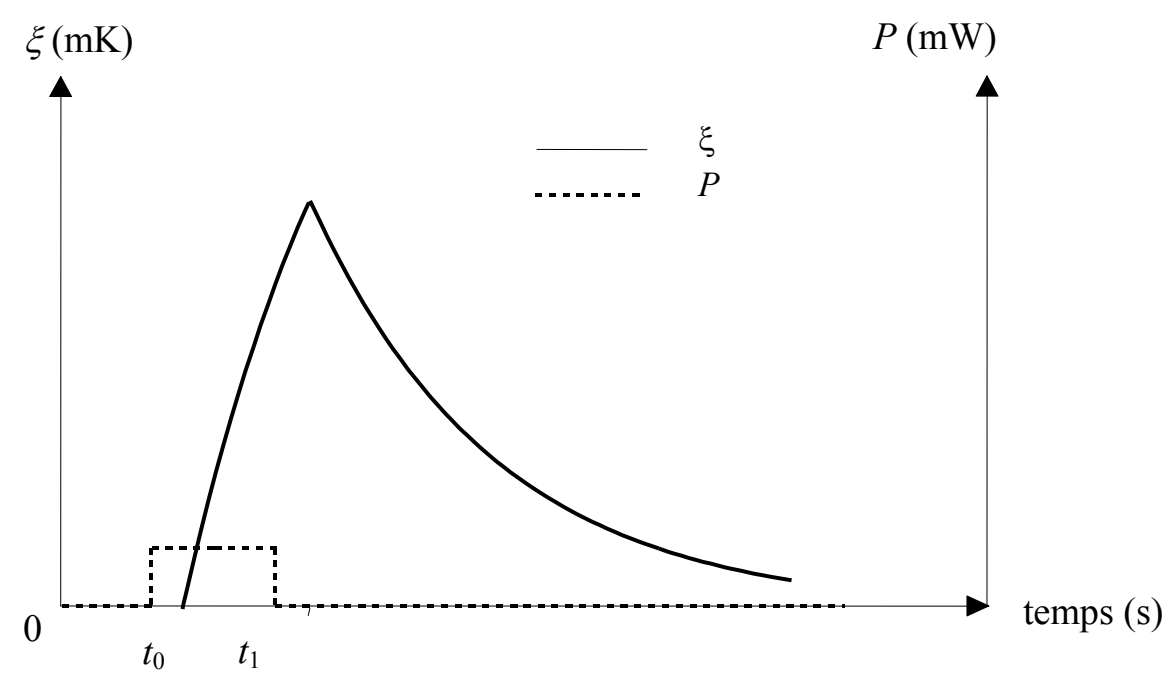

FIG. 2. Évolution thermique temporelle caractéristique d'un échantillon soumis à un échelon de puissance de rayonnement $P$. Les unités sont arbitraires, généralement exprimées en $\mathrm{mK}$ pour $\xi$, en $\mathrm{mW}$ pour $P$ et en seconde pour le temps.

L'échantillon est exposé à une puissance de rayonnement $P$ entre les temps $t_{0}$ et $t_{1}$. La figure $\underline{2}$ présente l'évolution caractéristique de l'écart de température $T_{\mathrm{e}}-T_{o}$ que l'on peut observer au cours du temps. L'évolution thermique temporelle s'obtient aisément en effectuant un bilan thermique élémentaire, dans l'hypothèse où le matériau est à température homogène ; expérimentalement ceci 
revient à réaliser un éclairement aussi uniforme que possible. On pose $\xi=T_{e}-T_{o}$; le bilan thermique conduit à l'équation :

$$
m c_{p} \frac{\mathrm{d} \xi}{\mathrm{d} t}+g \xi-\alpha P=0
$$

où $m$ est la masse de l'échantillon, $c_{p}$ sa chaleur spécifique, $\alpha$ la fraction de rayonnement absorbé, $P$ la puissance laser incidente appliquée entre $t_{0}$ et $t_{1}$ et $g$ un coefficient caractérisant les pertes thermiques vers le milieu ambiant que l'on suppose proportionnelles à $\xi$. Les solutions sont :

$$
\begin{array}{ll}
\xi(t)=0 & \text { pour } t \leq t_{0}, \\
\xi(t)=\frac{\alpha P}{g}\left[1-e^{-\frac{g}{m c_{p}}\left(t-t_{0}\right)}\right] & \text { pour } t_{0}<t<t_{1}, \\
\xi(t)=\xi\left(t_{1}\right) e^{-\frac{g}{m c_{p}}\left(t-t_{1}\right)} & \text { pour } t>t_{1} .
\end{array}
$$

\subsubsection{Analyse}

Plusieurs analyses de la réponse temporelle sont possibles afin d'en extraire la fraction de rayonnement absorbée $\alpha[1]$.

\subsubsection{Méthode du gradient thermique}

Pour une même valeur de $\xi$ on choisit sur la courbe de réponse (en général lissée) deux points $A$ et $B$ correspondant respectivement à la phase d'échauffement et à celle de refroidissement du matériau $\left(\xi\left(t_{c}\right)=\xi\left(t_{r}\right)\right)$, comme illustré figure $\underline{3}$. L'addition des pentes des tangentes en ces points permet de déterminer $\alpha$. En effet, d'après les équations précédentes on a : $\frac{\alpha P}{m c_{p}}=\frac{\mathrm{d} \xi}{\mathrm{d} t}\left(t_{c}\right)+\frac{g}{m c_{p}} \xi\left(t_{c}\right)$, avec $\frac{\mathrm{d} \xi}{\mathrm{d} t}\left(t_{r}\right)=-\frac{g}{m c_{p}} \xi\left(t_{r}\right)=-\frac{g}{m c_{p}} \xi\left(t_{c}\right) ;$ d'où $\frac{\alpha P}{m c_{p}}=\frac{\mathrm{d} \xi}{\mathrm{d} t}\left(t_{c}\right)-\frac{\mathrm{d} \xi}{\mathrm{d} t}\left(t_{r}\right)$.

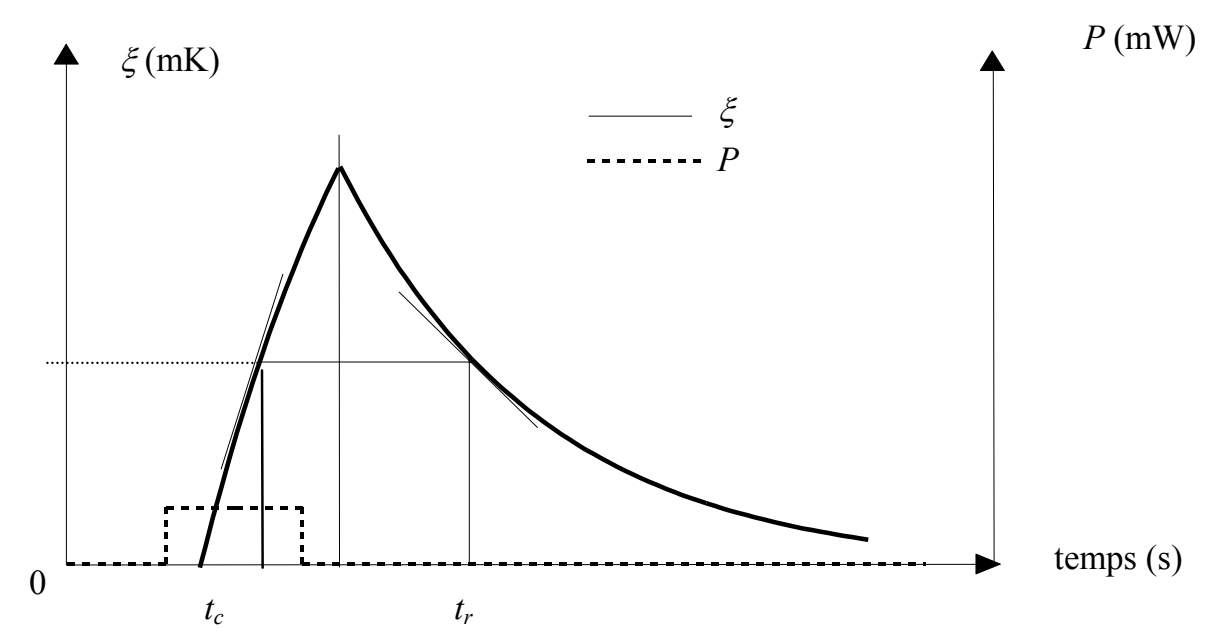

FIG. 3. Méthode du gradient thermique.

\subsubsection{Méthode du pulse}

Comme indiqué sur la figure $\underline{4}$, on extrapole la courbe de refroidissement jusqu'au temps $t=t_{0}+\frac{t_{p}}{2}=\frac{t_{1}-t_{0}}{2}$ pour obtenir l'écart de température $\xi_{\text {extr }}$, interprété comme représentant l'écart 
équivalent à celui dû à une énergie incidente instantanée $E_{p u l s e}=P t_{p}$. L'intérêt de la méthode apparaît pour les temps courts ou pour des faibles valeurs de $g$; on a alors :

$$
\xi_{\text {extr }}=\frac{\alpha P}{g}\left(1-e^{-\frac{g}{m c_{p}} t_{p}}\right) e^{\frac{g}{m c_{p}} \frac{t_{p}}{2}} \approx \frac{\alpha E_{\text {pulse }}}{m c_{p}}\left[1+\frac{\left(\frac{g}{m c_{p}} t_{p}\right)^{2}}{24}\right] .
$$

Pour $\left(\frac{g}{m c_{p}} t_{p}\right)^{2}$ très petit devant 1 on peut calculer directement $\alpha$ à partir de $\xi_{\text {extr. }}$.

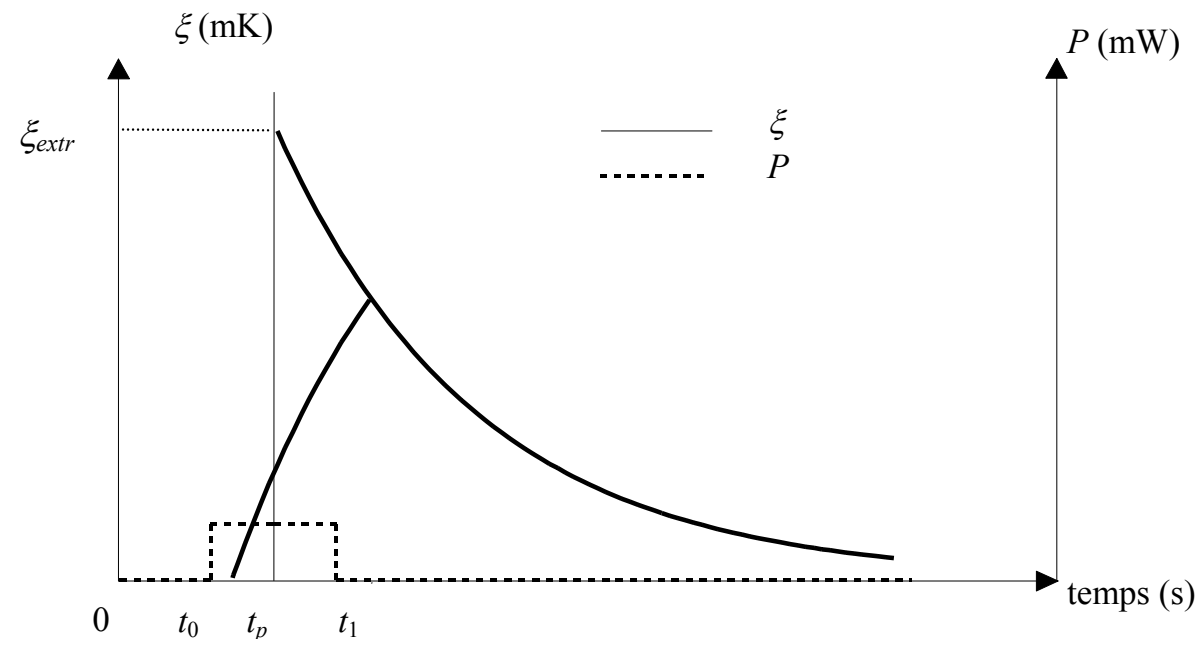

FIG. 4. Méthode du pulse.

\subsubsection{Méthode d'intégration}

On intègre l'équation de base entre deux temps $t_{i}$ et $t_{f}$. On obtient alors :

$$
\frac{\alpha P}{m c_{p}}\left(t_{f}-t_{i}\right)=\xi\left(t_{f}\right)-\xi\left(t_{i}\right)+\frac{g}{m c_{p}} \int_{t_{i}}^{t_{f}} \xi(t) \mathrm{d} t .
$$

Ayant déterminé $g$ par ailleurs, on mesure l'aire limitée par la courbe de réponse prise de $t_{i}$ à $t_{f}$, comme représenté sur la figure $\underline{5}$.

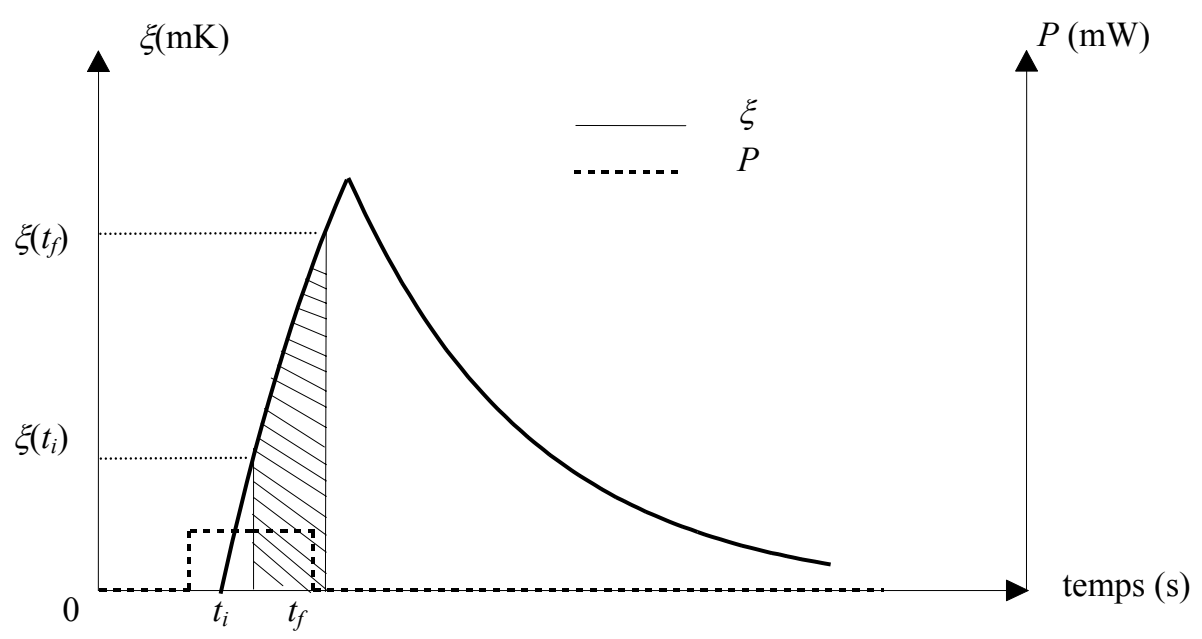

FIG. 5. Méthode d'intégration. 


\subsubsection{Ajustement exponentiel}

C'est une méthode générale : on ajuste les deux exponentielles de la courbe de réponse donnée sur la

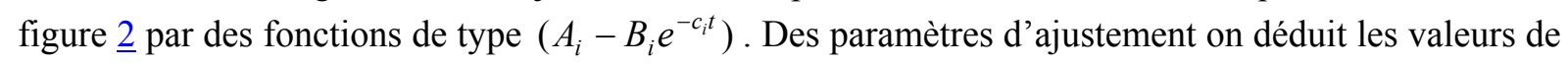
$\alpha$ et $\frac{g}{m c_{p}}$.

On soulignera que la meilleure précision est obtenue par la méthode du pulse dans les conditions d'utilisation mentionnées et par la méthode d'ajustement exponentiel pour les autres cas de figure. En fonction de l'appareillage développé la sensibilité de telles méthodes est de l'ordre de $100 \mathrm{ppm}$.

\subsection{Méthodes photo-acoustiques}

Le principe de ces techniques consiste à mesurer la modification d'une propriété physique du matériau sous l'effet d'un rayonnement (laser). Comme dans le cas des méthodes calorimétriques on s'arrangera expérimentalement pour que l'hypothèse de base soit toujours vérifiée, à savoir l'homogénéité de température dans l'échantillon.

\subsubsection{Détection pyroélectrique []]}

\subsubsection{Principe et montage expérimental}

La méthode est utilisable pour les cristaux polaires, présentant une modification de leur polarisation spontanée lors d'une variation de température. On enregistre alors le signal électrique fourni par l'échantillon monté en détecteur de type II (Fig. 6) et soumis à un rayonnement modulé comme indiqué figure $\underline{7}$. La réponse est fournie par une détection synchrone.

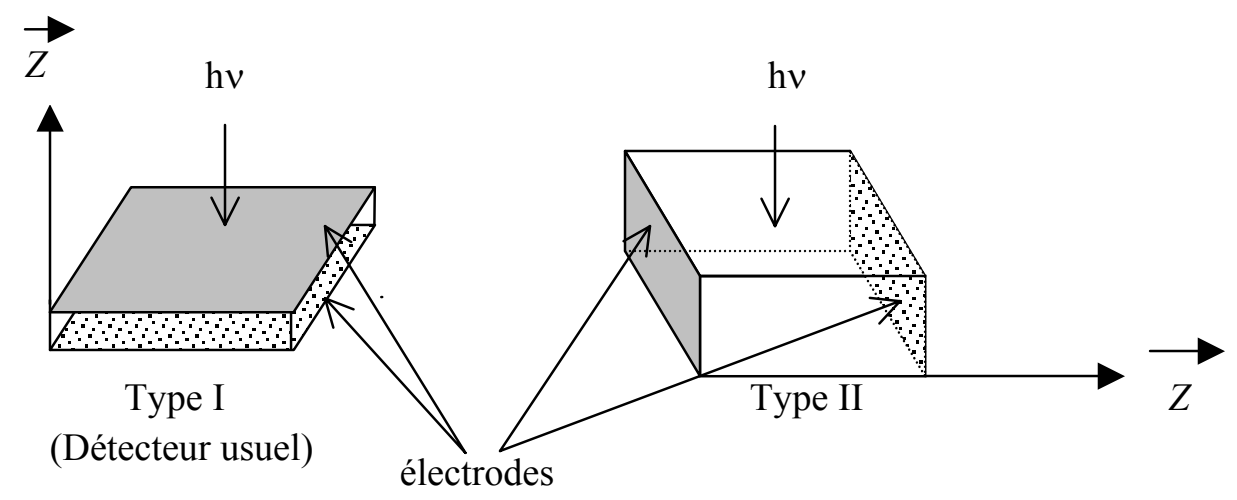

FIG. 6. Détection pyroélectrique de type I et II. Les électrodes sont déposées sur les faces polaires $(Z)$ des échantillons. Le cas I correspond aux détecteurs employés habituellement, où le rayonnement est absorbé directement sur une électrode $(\alpha=100 \%)$; le second met en jeu l'absorption propre du matériau.

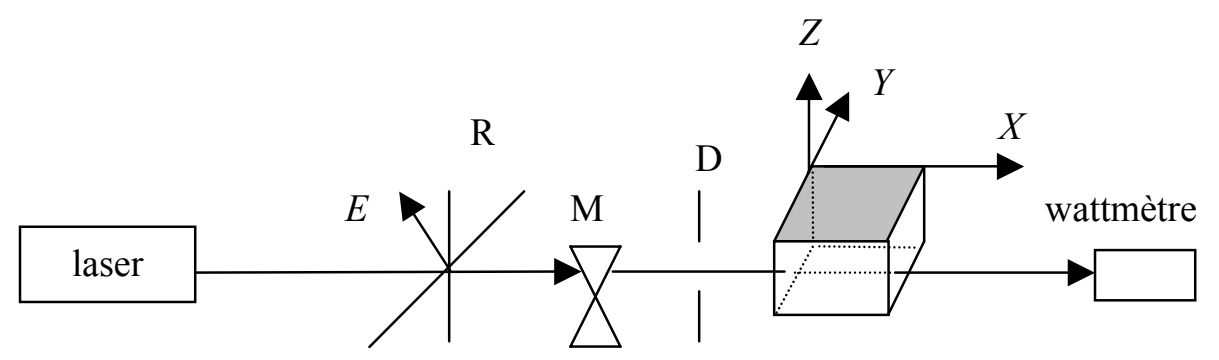

FIG. 7. Mesure de coefficients d'absorption par méthode pyroélectrique. Le rotateur de polarisation R placé devant le modulateur $\mathrm{M}$ et le diaphragme $\mathrm{D}$ permet d'ajuster la direction du champ $E$ du rayonnement incident par rapport aux axes cristallographiques $X, Y$ et $Z$ de l'échantillon. 


\subsubsection{Analyse}

Pour une modulation en carré on démontre que la réponse $V_{\text {eff }}$ détectée en phase est donnée par :

$$
V_{e f f}=\alpha \frac{2 \sqrt{2}}{\pi} R \omega p\left(1+\omega^{2} \tau_{t}{ }^{2}\right)^{-1 / 2}\left(1+\omega^{2} \tau_{e}{ }^{2}\right)^{-1 / 2} \Phi_{m},
$$

où $\alpha$ est la fraction de rayonnement absorbé et $\omega=2 \pi f$ la pulsation de la puissance modulée. $\Phi_{m}$ est la puissance moyenne incidente. $\tau_{\mathrm{e}}$ et $\tau_{\mathrm{t}}$ sont les constantes de temps électrique et thermique; $\tau_{e}=R C$ où $R$ et $C$ sont la résistance électrique et la capacité du cristal muni de ses connexions, mesurées à la fréquence $f$ avec un pont d'impédance, $\tau_{t}=C / G$ où $C$ et $G$ représentent la capacité et la conductivité thermique du dispositif. $p=\frac{\mathrm{d} P_{s}}{\mathrm{~d} T}$ est le coefficient pyroélectrique, $P_{s}$ étant la polarisation spontanée du matériau.

Le coefficient d'absorption $K\left(\mathrm{~cm}^{-1}\right)$ est relié à $\alpha$ par la relation :

$$
K=-\frac{1}{d} \log \left[1-\frac{\alpha}{1-R}\right]
$$

où $d$ est l'épaisseur de l'échantillon et $R=\frac{(n-1)^{2}}{(n+1)^{2}}$ son coefficient de réflexion. Si on choisit $\omega$ tel que $\omega^{2} \tau_{t}^{2}>>1, \alpha$ sera donné par :

$$
\alpha=\frac{\pi}{2 \sqrt{2}} \frac{\tau_{t}}{p R}\left(1+\omega^{2} \tau_{e}^{2}\right)^{1 / 2} \frac{V_{e f f}}{\Phi_{m}} .
$$

C'est ce qui est réalisé en pratique.

Cette méthode est d'une grande sensibilité si $p$ est important mais nécessite de s'assurer que le cristal examiné est monodomaine et exempt de centres diffusants. Elle peut aussi être employée dans le cas de matériaux semiconducteurs ou conducteurs ioniques à condition d'évaluer auparavant leur coefficient pyroélectrique par la méthode dynamique où on compare leur réponse à celle d'un matériau de référence, les deux étant montés en détecteurs de type I.

\subsubsection{Bolométrie piézo-électrique [3]}

\subsubsection{Principe et montage expérimental}

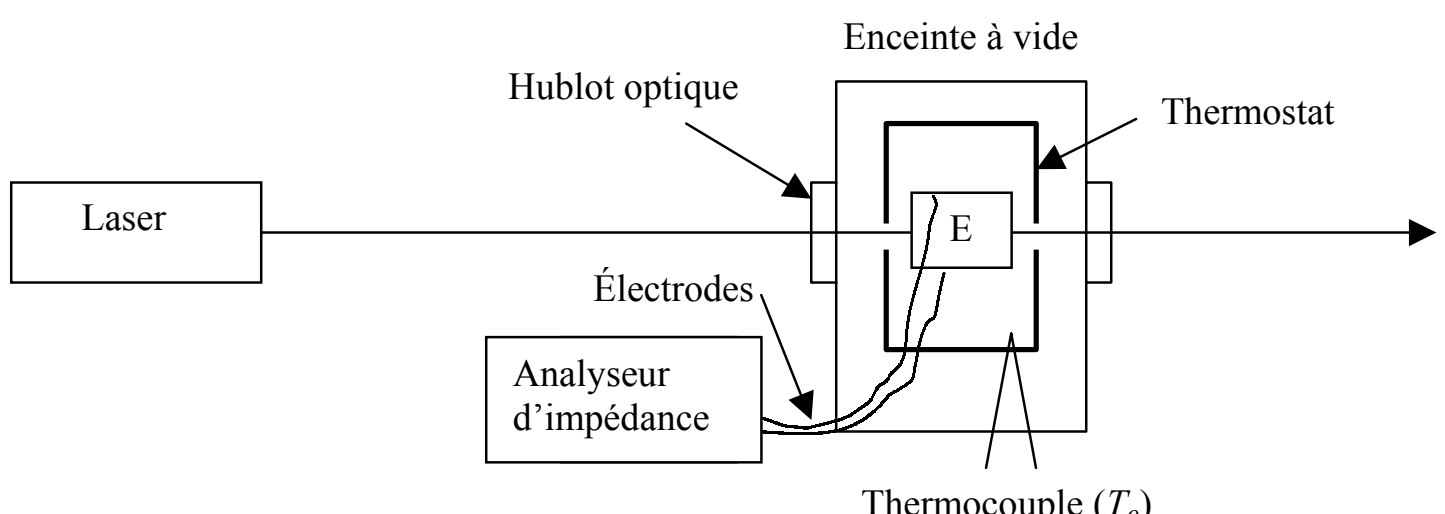

FIG. 8. Bolométrie piézo-électrique. L’échantillon E muni d'électrodes est placé dans un thermostat régulé à la température $T_{o}$, à l'intérieur d'une enceinte à vide. La dérive thermique d'une fréquence de résonance sélectionnée est analysée au moyen d'un pont d'impédance.

Cette méthode proposée récemment est utilisable pour les matériaux appartenant aux classes cristallines susceptibles de présenter cet effet. On choisit une résonance piézo-électrique simple du 
matériau, dont la fréquence se trouvera modifiée par l'élévation de température induite par un rayonnement laser. Un montage d'expérimentation type est donné figure $\underline{8}$.

Dans le modèle dit de l'aiguille, à une température $T$, la fréquence de résonance $f_{r}$ est donnée par $f_{r}=(2 L \sqrt{\rho s})^{-1}$ où $L$ est la longueur du cristal, $\rho$ sa densité et $s$ le coefficient d'élasticité [4].

\subsubsection{Méthodologie d'étude : exemple de $\mathrm{LiNbO}_{3}$}

Pour décrire les différentes étapes nécessaires à la mise en œuvre de la méthode on s'intéressera au cas concret d'un échantillon parallélépipédique de niobate de lithium, orienté par rapport aux axes cristallographiques $X, Y$ et $Z$ comme indiqué sur la figure $\underline{9}$.

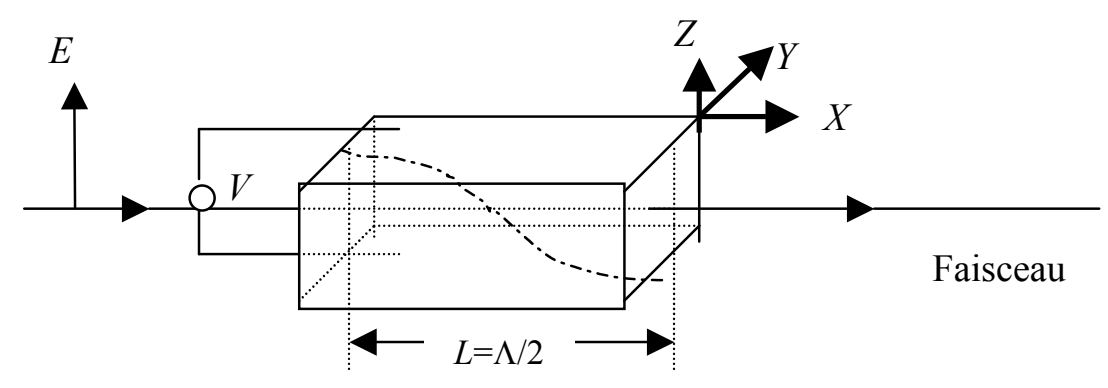

FIG. 9. Bolométrie piézo-électrique. Pour exemple, un échantillon parallélépipédique de $\mathrm{LiNbO}_{3}$, de dimension $8 \times 4 \times 4 \mathrm{~mm}^{3}$ est muni d'électrodes sur les faces $Z$. Une tension alternative $V$ d'environ 1 volt, appliquée à la fréquence de résonance $f_{r}$, engendre une onde stationnaire dont l'amplitude est visualisée par la courbe dessinée au centre du schéma. Cette amplitude est maximum au niveau des faces optiques $X$ du cristal et minimum au centre. Pour le champ de contraintes, c'est l'inverse. On a sélectionné un mode d'extension simple correspondant à une longueur de cristal égale à la moitié de la longueur d'onde acoustique $\Lambda$. après :

La méthodologie d'étude est scindée en cinq étapes, décrites dans les paragraphes a) à e) ci-

a) en l'absence de rayonnement laser, balayage en fréquence avec le pont d'impédance pour identifier le mode fondamental d'extension de plus basse fréquence. Dans cette configuration il correspond à la fréquence $f_{r}=\left(2 L \sqrt{\rho s_{11}}\right)^{-1}$.

b) évaluation de $\frac{\mathrm{d} f_{r}}{\mathrm{~d} T}$ : on démontre d'abord que $\frac{\mathrm{d} f_{r}}{\mathrm{~d} T}=\frac{1}{2} f_{r}\left[\alpha_{z}-\frac{1}{s_{11}} \frac{\mathrm{d} s_{11}}{\mathrm{~d} T}\right]$, paramètre que l'on calcule ensuite connaissant le coefficient $\alpha_{Z}$ de dilatation linéaire suivant $Z$ et $\frac{\mathrm{d} s_{11}}{\mathrm{~d} T}$. Dans l'exemple cité on trouve $-30 \mathrm{~Hz} /{ }^{\circ} \mathrm{C}$.

c) détermination de $f_{r}(T)$ : pour plusieurs valeurs fixées de la température $T$, établies par contrôle du thermostat, on effectue un balayage en fréquence à l'aide du pont d'impédance. On relève les courbes de conductivité et de capacité électriques $S(T)$ et $C(T)$, dont les allures générales sont indiquées sur la figure $\underline{10}$.

d) les pics de conductivité $S$ sont ajustés par une loi de Lorentz de la forme :

$$
S=S_{0} \frac{f^{2}(\Delta f)^{2}}{\left(f^{2}-f_{r}^{2}\right)^{2}+f^{2}(\Delta f)^{2}},
$$

où $\Delta f$ est la largeur du pic à mi-hauteur. La procédure d'ajustement conduit à une détermination des fréquences de résonance à $\pm 1 \mathrm{~Hz}$ près. Une courbe typique de $f_{r}$ en fonction de la température est donnée sur la figure $\underline{11}$. Pour l'exemple indiqué la loi de variation linéaire donne $\frac{\mathrm{d} f_{r}}{\mathrm{~d} T}=-32 \mathrm{~Hz} /{ }^{\circ} \mathrm{C}$; en comparant cette valeur avec le résultat $\mathrm{du}$ 
paragraphe b) on peut en déduire que le modèle de l'aiguille est approprié. À ce stade l'échantillon se trouve « calibré ».

e) détermination du shift en fréquence $\Delta f_{r}$ induit sous rayonnement laser de puissance $P$ connue : une fois l'équilibre thermique atteint on en déduit aisément l'augmentation de température $\Delta T$ correspondante subie par l'échantillon.

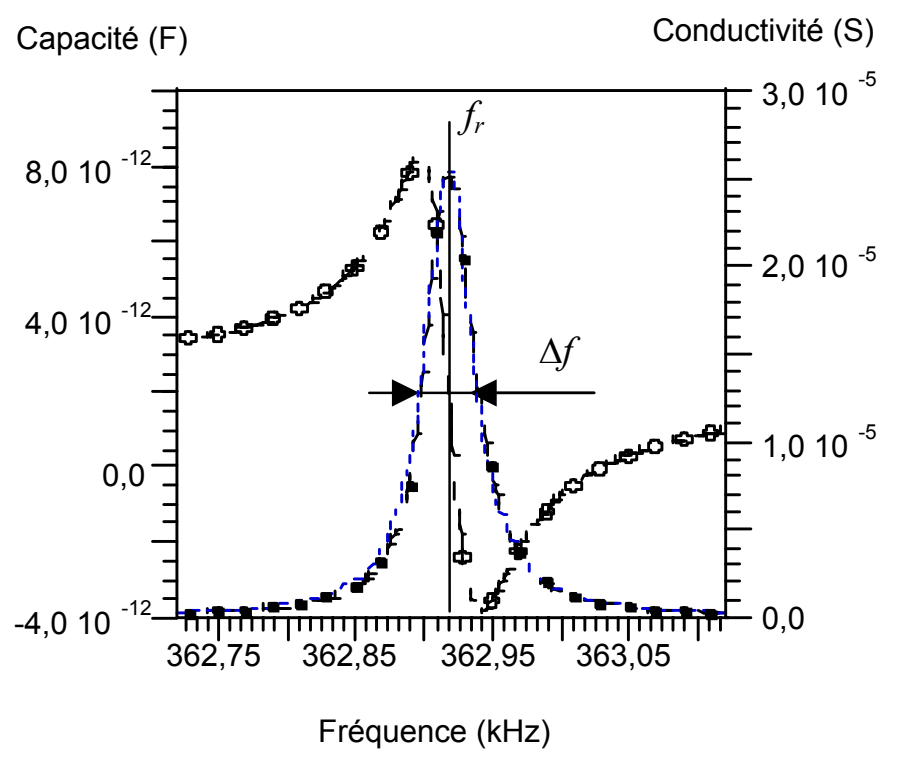

FIG. 10. Paramètres électriques de la résonance piézo-électrique. Le pic de conductivité situe la fréquence de résonance $f_{r}$. $\Delta f$ est la largeur à mi-hauteur.

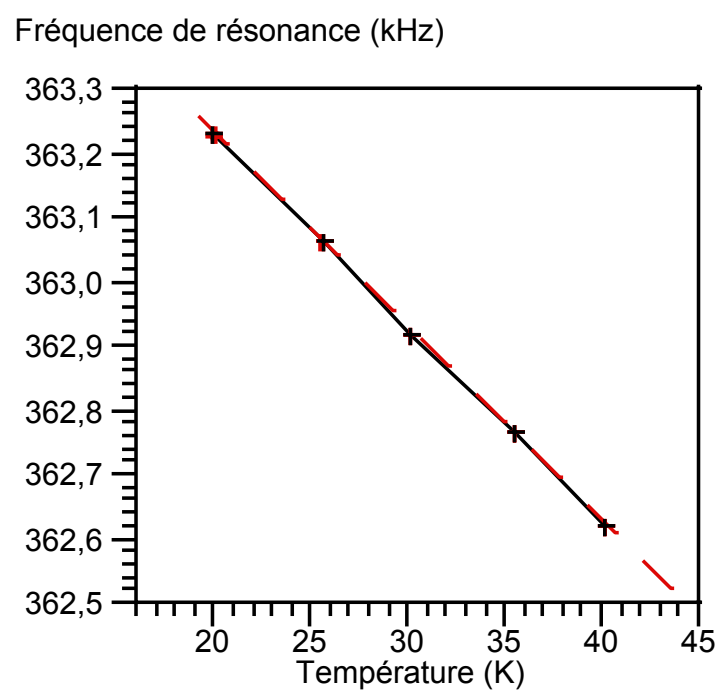

FIG. 11. Évolution thermique d'une fréquence de résonance piézo-électrique.

Remarque sur la précision : l'incertitude de $1 \mathrm{~Hz}$ sur la détermination de $f_{r}$ conduit à une incertitude de $60 \mathrm{mK}$ sur celle de $\Delta T$. L'utilisation de la conductivité électrique pour obtenir la fréquence de résonance prise comme repère thermométrique est d'une grande sensibilité mais de précision moyenne. Par contre on notera que le changement de phase de $\pi$ observé sur la courbe $C(f)$ au voisinage de $f_{r}$ se traduira en fait par une valeur élevée de $\frac{\mathrm{d} C}{\mathrm{~d} T}$. La capacité se trouve donc être le paramètre pertinent pour évaluer $\Delta T$ et non la fréquence de résonance. 
Dans l'exemple proposé un ajustement linéaire du comportement de la capacité au voisinage de la fréquence de $f_{r}$ donne $\frac{\mathrm{d} C}{\mathrm{~d} T}=44,81 \mathrm{pF} / \mathrm{K}$. La résolution d'un pont d'impédance de bonne qualité est de l'ordre de $0,01 \mathrm{pF}$; ceci correspondra alors à une résolution de $4.10^{-4} \mathrm{~K}$ pour la mesure de $\Delta T$.

\subsubsection{Détermination du coefficient d'absorption}

\subsection{Bilan thermique : équation bolométrique}

Soit $T_{o}$ la température correspondant à l'état initial, en l'absence de flux lumineux. $T_{o}$ est la température d'ambiance fixée par le thermostat. On pose $T=T_{o}+\xi$, température de l'échantillon soumis à l'éclairement $\Phi_{o}$. Suivant la loi de Beer-Lambert $\left(I=I_{0} e^{-k L}\right)$ et compte tenu des réflexions multiples, le flux $\Phi_{a}$ absorbé par le cristal est donné par :

$$
\Phi_{a}=\Phi_{0} \frac{(1-R)\left(1-e^{-k L}\right)}{1-R e^{-k L}}
$$

où $R$ est le coefficient de réflexion du matériau et $k$ son coefficient d'absorption.

Le bilan thermique effectué sur l'intervalle de temps $d t$ donne :

- $\quad$ flux $\mathrm{d} \Phi_{a}$ absorbé par le cristal : $\mathrm{d} \Phi_{a}=\Phi_{a} \mathrm{~d} t$;

- flux $\mathrm{d} \Phi_{t h}$ provenant du thermostat : $\mathrm{d} \Phi_{a}=\sigma A T_{0}{ }^{4} \mathrm{~d} t$ (loi de Stefan); $A$ est la surface de l'échantillon en regard avec le thermostat et $\sigma$ la constante de Stefan ;

- augmentation $\mathrm{d} u$ d'énergie interne $: \mathrm{d} u=m c_{p} \frac{\mathrm{d} \xi}{\mathrm{d} t} \mathrm{~d} t ; m$ et $c_{p}$ sont la masse et la chaleur spécifique de l'élément ;

- pertes par rayonnement vers le milieu ambiant : $\mathrm{d} \Phi_{e}=\sigma A T^{4} \mathrm{~d} t$;

- pertes par conduction $\mathrm{d} \Phi_{c}$ : elles sont dues au support échantillon et à la « qualité » du vide dans l'enceinte, ce qui est difficile d'évaluer de façon précise. De façon habituelle on les suppose proportionnelles à l'écart de température $T-T_{o}: \mathrm{d} \Phi_{c}=g\left(T-T_{o}\right), g$ caractérisant la conduction thermique globale vers le thermostat.

L'équation bolométrique s'écrit : $\mathrm{d} \Phi_{a}+\mathrm{d} \Phi_{t h}=\mathrm{d} \Phi_{e}+\mathrm{d} u+\mathrm{d} \Phi_{c}$, soit :

$$
m c_{p} \frac{\mathrm{d} \xi}{\mathrm{d} t}+\left(g+4 \sigma A T_{0}^{3}\right) \xi-\alpha \Phi_{0}=0
$$

où l'on a posé $\alpha=\frac{(1-R)\left(1-e^{-k L}\right)}{1-R e^{-k L}}$ et, tenu compte de $\left(T_{0}^{4}-T^{4}\right) \simeq-4 T_{0}^{3} \xi$, la solution est de la forme $\xi=\xi_{0}\left(1-e^{-\frac{t}{\tau}}\right)$, avec $\xi_{0}=\frac{\alpha \Phi_{0}}{g+4 \sigma A T_{0}{ }^{3}}$ et $\tau=\frac{m c}{g+4 \sigma A T_{0}{ }^{3}}$.

\subsection{Calcul du coefficient d'absorption $\mathrm{k}$}

Les formules précédentes donnent : $\alpha=\frac{m c_{p} \xi_{0}}{\tau \Phi_{0}}$; et on a $k=-\frac{1}{L} \log \frac{R+\alpha-1}{R(\alpha+1)-1}$.

L'ajustement de l'évolution de $C$ en fonction du temps permet d'en déduire la constante de temps $\tau$, l'amplitude de l'effet thermique $\Delta C$ donnant $\xi_{0}$. On mesure $\Phi_{0}$ et on détermine $\tau$ et $\xi_{0}$; ces deux valeurs conduisent à la valeur de $\alpha$ et donc de $k$. La figure 12 illustre l'exemple cité. 
La sensibilité de cette méthode est voisine de $1 \mathrm{ppm}$ et la précision de l'ordre de $10 \%$. Comme toutes les techniques de type photo-acoustique elle nécessite cependant une minutie expérimentale très spécifique.

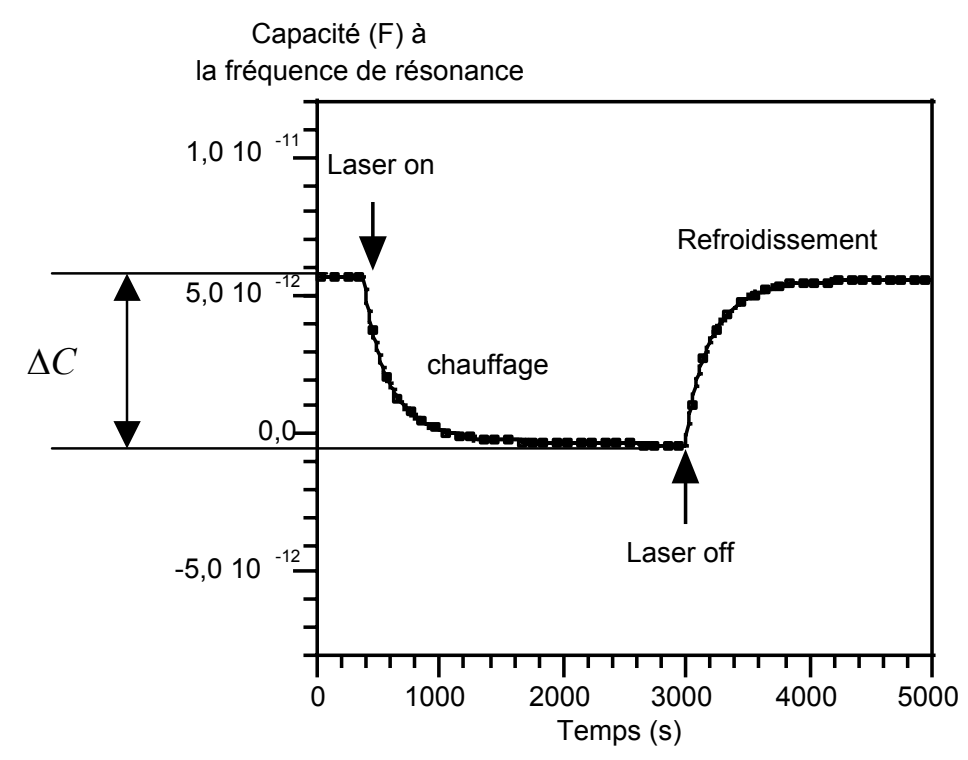

FIG. 12. Bolométrie piézo-électrique : évolution temporelle de la capacité observée à la résonance.

\section{COEFFICIENTS THERMO-OPTIQUES}

\subsection{Définition}

Ces coefficients caractérisent, à une longueur d'onde donnée, la variation d'indice(s) de réfraction $\mathrm{d} n / \mathrm{d} T$ d'un matériau en fonction de sa température.

\subsection{Techniques de mesure}

\subsubsection{Méthode du prisme}

Plusieurs géométries utilisant la réfraction par un prisme permettent d'accéder à la valeur de l'indice $n$ d'un matériau: méthode d'Arnulf, autocollimation sur la face interne d'un prisme à angle droit, mesure du minimum de déviation... La figure $\underline{13}$ représente cette dernière méthode bien connue, pour laquelle on a :

$$
n=\frac{\sin \frac{A+D_{m}}{2}}{\sin \frac{A}{2}} .
$$

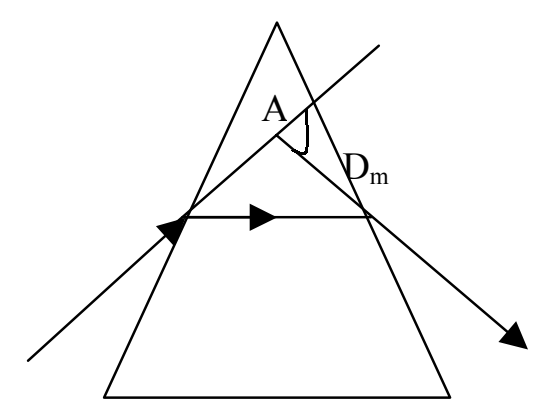

FIG. 13. Méthode du prisme au minimum de déviation. 
Dans tous les cas la détermination de $n(T)$ à une longueur d'onde est réalisée en thermostatant le prisme à plusieurs valeurs de la température, choisies dans l'intervalle que l'on veut explorer. Les principaux problèmes inhérents à ce type de technique sont liés à la dimension relativement importante requise pour les échantillons. Celle-ci entraîne quelques difficultés pour obtenir une bonne stabilité de la température, la présence de gradients à l'intérieur du matériau et des constantes de temps thermiques élevées. On notera par ailleurs qu'une absorption non négligeable aura pour effet de modifier la symétrie d'intensité transverse du faisceau lumineux.

\subsubsection{Méthodes interférométriques}

Le principe de telles méthodes est de déterminer la modification d'épaisseur optique d'un matériau engendrée par une variation de sa température. Deux mises en œuvre expérimentales simples sont possibles :

- dans un interféromètre à deux ondes, de type Michelson ou Mach-Zehnder par exemple, on interpose sur l'un des deux bras un échantillon placé dans une enceinte à température contrôlable. On enregistre alors le nombre de franges qui se déplacent lorsque l'on fait varier la température du matériau sur un intervalle de température $\Delta T$ choisi.

- si l'échantillon comporte deux faces optiques planes et parallèles il constitue lui-même un interféromètre de Fabry-Pérot à ondes multiples. On opèrera de la même façon que ci-dessus pour des franges observées soit en transmission, soit en réflexion.

Dans ces techniques le déplacement de franges enregistré correspond à une variation d'épaisseur optique :

$$
\Delta(n e)=p \frac{\lambda}{2}+k
$$

$\lambda$ étant la longueur d'onde du rayonnement utilisé, $p$ un nombre entier et $k$ l'excédent fractionnaire. On remarquera qu'il faut donc aussi connaître le coefficient de dilatation pour pouvoir déterminer le coefficient thermo-optique. Ces deux mesures peuvent être réalisées simultanément au moyen du dilatomètre interférométrique absolu décrit au paragraphe $\underline{6}$.

\section{COEFFICIENTS PIÉZOÉLECTRIQUES}

\subsection{Effet piézo-électrique : rappels []]}

Effet direct : si une contrainte $\sigma$ est appliquée à un cristal, la polarisation $P$ engendrée par unité de surface est: $P_{i j k}=d_{i j k} \sigma_{j k}$, où $\sigma_{j k}$ sont les modules piézo-électriques. En général $d_{i j k}=d_{i k j}$ (indiscernabilité). En notation matricielle : $P_{i}=d_{i j} \sigma_{j}$, avec $i=1,2,3$ et $j=1,2, \ldots .6$. Si le cristal possède en outre une polarisation spontanée (cas des ferroélectriques par exemple), $P_{i}$ représente le changement de celle-ci dû à la contrainte appliquée.

Effet inverse : pour un champ électrique appliqué $E$, le tenseur des déformations s'écrit : $\varepsilon_{j k}=d_{i j k} E_{i}$, ou en notation matricielle $\varepsilon_{j}=d_{i j} E_{i}$, avec $i=1,2,3$ et $j=1,2, \ldots 6$.

On trouvera par ailleurs la forme de ces matrices et les coefficients non nuls établis en fonction des éléments de symétrie propres à chacune des 20 classes de symétrie piézoélectriques.

Les déplacements $u_{i}$ engendrés par cet effet sont donnés par $u_{i}=\varepsilon_{i j} e_{j}$, où $e_{j}$ est la dimension du cristal dans la direction $j$. À titre d'exemple le tableau I donne la matrice des modules piézoélectriques dans le cas du système orthorhombique mm2 où 5 éléments sont non nuls. 


$\begin{array}{llllll}0 & 0 & 0 & 0 & d_{15} & 0 \\ 0 & 0 & 0 & d_{24} & 0 & 0 \\ d_{31} & d_{32} & d_{33} & 0 & 0 & 0\end{array}$

Tableau I. Modules piézo-électriques pour la classe de symétrie mm2.

Dans cet exemple un champ appliqué $E$, de composantes $E_{1}, E_{2}$ et $E_{3}$, donnera lieu aux déformations :

$$
\varepsilon_{1}=d_{31} E_{3}, \varepsilon_{2}=d_{32} E_{3}, \varepsilon_{3}=d_{33} E_{3}, \varepsilon_{4}=d_{24} E_{2} \text { et } \varepsilon_{5}=d_{15} E_{1} .
$$

Un cas concret peut être illustré par un cristal parallélépipédique, taillé suivant les directions principales 1,2 et 3 , de dimensions respectives $e_{x}$, $e_{y}$ et $e_{z}$ suivant ces directions. Si $E \equiv E_{3}$, la déformation engendrée suivant la direction 1 sera donnée par $u_{1}=d_{31} E_{3} e_{x}$.

\subsection{Méthodes de mesure}

On détermine les modules piézoélectriques en utilisant soit l'effet direct, soit l'effet inverse.

Dans le premier cas on mesurera par exemple la polarisation induite par une contrainte connue, appliquée suivant une direction choisie et en collectant les charges apparues aux bornes d'un électromètre (méthode d'intégration des charges).

Dans le second cas on applique un champ $E$ connu et toute technique de mesure précise des déplacements engendrés est appropriée. On citera en particulier la mesure de la valeur de la capacité d'un condensateur, modifiée par le déplacement d'une de ses électrodes et l'interférométrie optique. Le dilatomètre absolu décrit paragraphe $\underline{6}$ est adapté aussi à ce type de mesure.

\section{COEFFICIENTS ÉLECTRO-OPTIQUES [ㅁ]}

\subsection{Effet électro-optique}

L'effet électro-optique concerne la variation des indices de réfraction d'un cristal soumis à l'action d'un champ électrique.

Pour un cristal l'ellipsoïde des indices rapporté à ses axes principaux $x, y$ et $z$ a pour équation :

$$
\frac{x^{2}}{n_{x}^{2}}+\frac{y^{2}}{n_{y}^{2}}+\frac{z^{2}}{n_{z}^{2}}=1
$$

Sous l'action d'un champ électrique appliqué $E$, cette équation devient au premier ordre (effet Pockels) :

$$
\left(\frac{1}{n_{x}{ }^{2}}+r_{1 k} E_{k}\right) x^{2}+\left(\frac{1}{n_{y}{ }^{2}}+r_{2 k} E_{k}\right) y^{2}+\left(\frac{1}{n_{z}{ }^{2}}+r_{3 k} E_{k}\right) z^{2}+2 y z r_{4 k} E_{k}+2 z x r_{5 k} E_{k}+2 x y r_{6 k} E_{k}=1 .
$$

Les $r_{i k}$ avec $i=1,2,3,4,5,6$ et $k=1,2,3$ sont les coefficients électro-optiques linéaires. La formule se lit avec la convention habituelle de sommation sur les indices $k$ répétés. On ne tient pas compte de l'effet du second ordre (effet Kerr). La représentation matricielle des $r_{i k}$ s'écrit de façon analogue à celle des coefficients piézoélectriques.

\subsection{Détermination des $r_{i k}$}

\subsubsection{Méthode ellipsométrique}

Dans les méthodes ellipsométriques l'échantillon est placé entre un polariseur et un analyseur. La figure 14 schématise une configuration possible (compensateur de Sénarmont). 


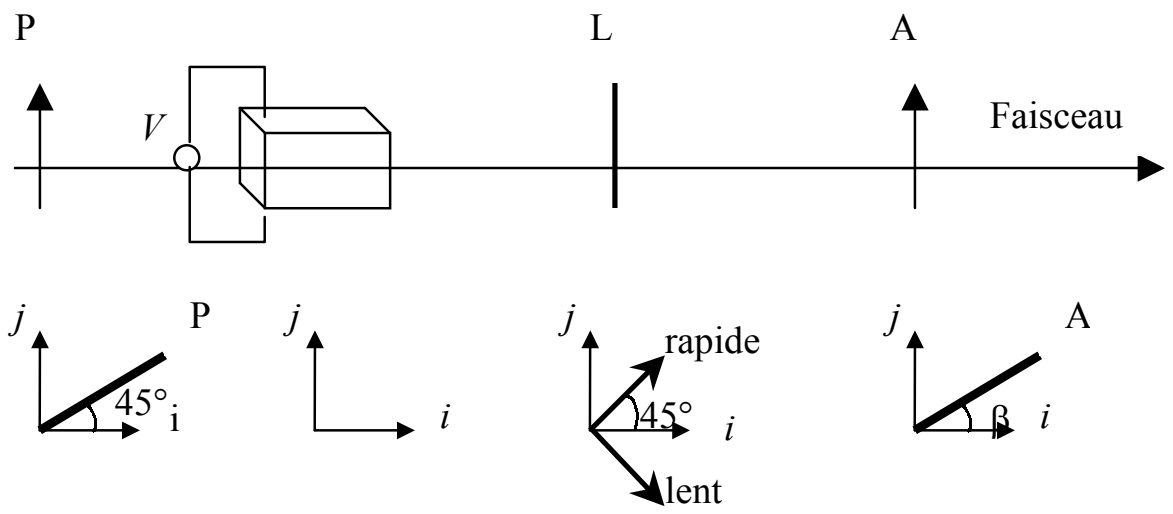

FIG. 14. Mesure de coefficients électro-optiques par ellipsométrie. $i$ et $j$ représentent deux directions principales de l'échantillon par rapport auxquelles sont repérées les orientations du polariseur $\mathrm{P}$, de la lame quart d'onde $\mathrm{L}$ et de l'analyseur A.

L'intensité transmise par le système est donnée par $I=\frac{I_{0}}{2}[1-\sin (\Gamma-2 \beta)]$, où $I_{0}$ est l'intensité incidente et $\beta$ l'angle entre l'axe $x$ et la direction de l'analyseur A. $\Gamma$ est le retard de phase entre les ondes principales : $\Gamma=\left(n_{i}-n_{j}\right) \frac{\omega}{c} e \cdot n_{i}$ et $n_{j}$ sont deux indices principaux du matériau et $e$ l'épaisseur traversée. Pour un champ appliqué $E$ de composantes $E_{l}(l=1,2$ ou 3), il peut s'écrire comme la somme de deux termes respectivement dus à la biréfringence naturelle et à la contribution électrooptique propre :

$$
\Gamma(E)=\left[n_{i}(E)-n_{j}(E)\right] \frac{\omega}{c} e=\left[n_{i}(0)-n_{j}(0)\right] \frac{\omega}{c} e+\frac{1}{2} n_{j}^{3}\left[r_{j l}-\left(\frac{n_{i}}{n_{j}}\right)^{3} r_{i l}\right] E_{l} \frac{\omega}{c} e=\Gamma(0)+\Gamma(E),
$$

où la variation de phase $\Gamma(E)$ s'exprime habituellement en posant $r_{c}=\left[r_{j l}-\left(\frac{n_{i}}{n_{j}}\right)^{3} r_{i l}\right]$.

On trouvera en référence [7] une description détaillée de plusieurs méthodes ellipsométriques et une évaluation des incertitudes de mesure en référence [8].

\subsubsection{Interférométrie}

Les méthodes interférométriques sont avantageusement utilisées lorsque la direction du champ appliqué n'entraîne pas de rotation de l'ellipsoïde des indices. Comme pour la détermination des coefficients thermo-optiques elles peuvent être de deux types.

\subsubsection{Interférométrie de Mach-Zehnder à deux ondes}

L'échantillon E est inséré dans l'un des deux bras de l'interféromètre comme schématisé figure 15 . L'application d'un champ électrique a pour effet de modifier la différence de phase entre les deux bras de l'interféromètre ce qui engendre une modification de la figure d'interférence, la température de l'échantillon étant parfaitement stable. On se reportera à la référence [9] pour plus de détails. 


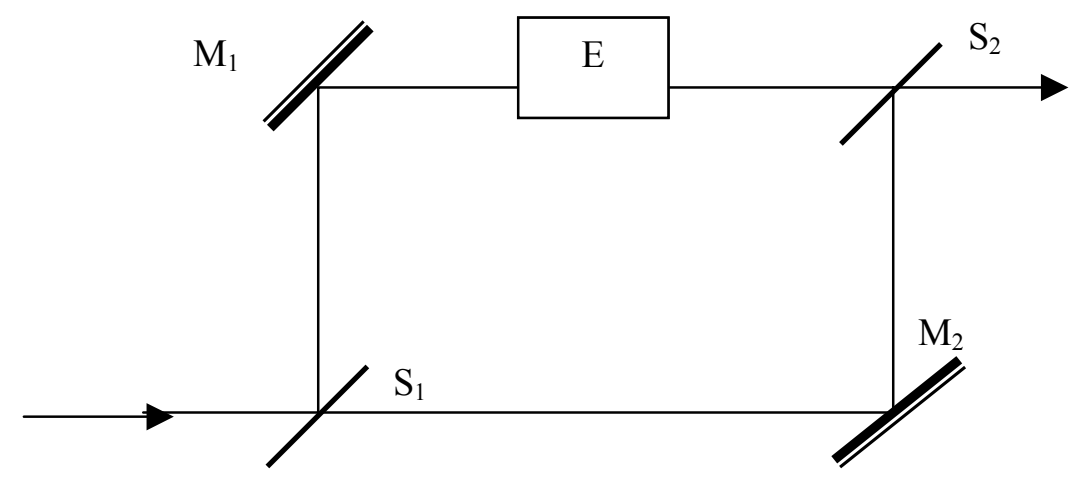

FIG. 15. Interférométrie de Mach-Zehnder pour la détermination de coefficients électro-optiques.

\subsubsection{Interférométrie de Fabry-Pérot à balayage thermique}

\subsection{Principe}

Les mesures de coefficients électro-optiques au moyen des techniques mentionnées ci-dessus demandent avant tout, pour être très précises, un contrôle rigoureux de la stabilité de température de l'échantillon. En effet, une dérive fortuite de celle-ci conduira à une variation d'indice par effet thermo-optique et pourra affecter la mesure d'une erreur difficilement quantifiable. La méthode interférométrique à ondes multiples décrite ci-après évite cet inconvénient en tirant avantage d'une dérive thermique contrôlée du matériau.

Dans cette méthode l'échantillon à faces planes et parallèles joue le rôle d'un interféromètre de Fabry-Pérot, comme dans le cas de la mesure des coefficients thermo-optiques. Les franges sont observées en réflexion et leur modification sous l'effet d'un champ électrique appliqué est comparée à la variation équivalente d'épaisseur optique obtenue lors d'une variation de température effectuée à champ nul. Dans la pratique ceci revient à enregistrer le défilement de franges lors d'une mesure de coefficient thermo-optique et en détectant simultanément le signal électro-optique au cours de la variation de température imposée à l'échantillon, ce que permet de réaliser l'interféromètre décrit au paragraphe $\underline{6}$. Dans cette technique dite de Fabry-Pérot à balayage thermique (méthode FPTSI), l'application d'un champ électrique alternatif réalise en fait une modulation de la phase $\phi(T)$ sur le système de franges généré par effet thermo-optique. Le signal électro-optique détecté en phase avec la modulation du champ, est alors proportionnel à la dérivée de la fonction d'Airy enregistrée lors de la variation de température de l'échantillon qui induit une variation de la phase $\phi(T)$. La figure $\underline{16}$ donne de façon qualitative la fonction d'Airy $R(\phi)$, à partir de laquelle on détermine le coefficient thermooptique, ainsi que la courbe représentant le module de sa dérivée $G(\phi)$ à partir de laquelle on détermine le coefficient électro-optique.

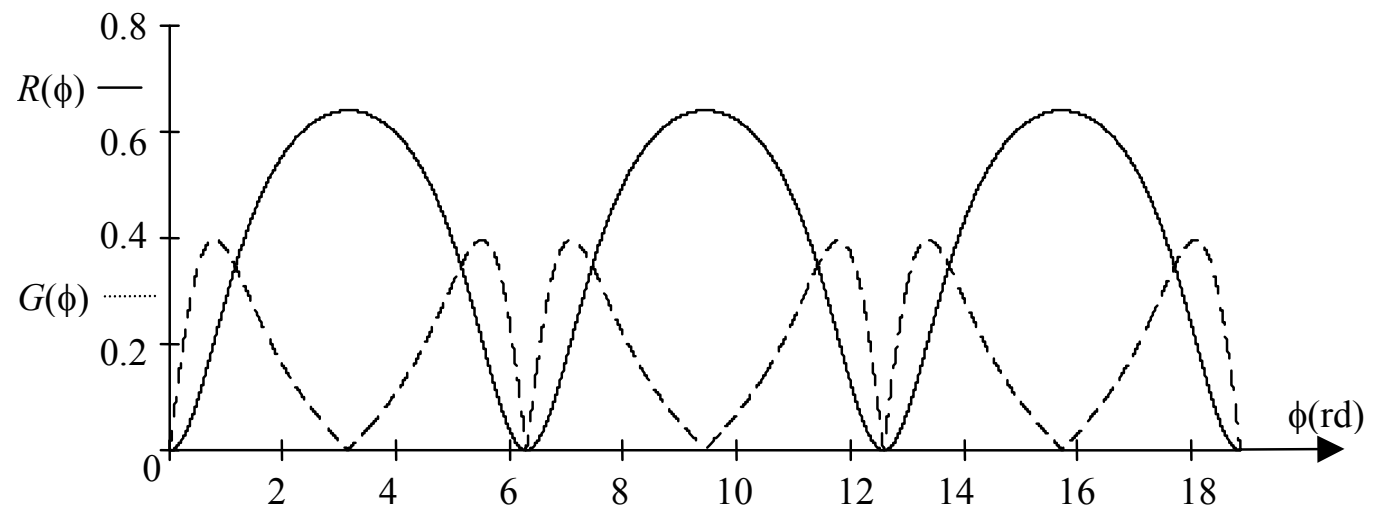

FIG. 16. Interférométrie de Fabry-Pérot à balayage thermique. La fonction $R(\phi)$ donnera le coefficient thermooptique et sa dérivée $G(\phi)$ le coefficient électro-optique ; l'indice de réfraction $n$ est supposé égal à 3 . 


\subsection{Exemple: cas du système $m m 2$}

Pour illustrer la méthodologie expérimentale on reprendra le cas du système orthorhombique $\mathrm{mm} 2$, pour lequel la forme du tenseur des coefficients électro-optiques est identique à celle décrite dans le paragraphe 4.1. Pour la géométrie qui y est mentionnée l'application d'un champ $E_{3}$ suivant l'axe $Z$ ne modifiera pas l'orientation de l'ellipsoïde des indices et les nouvelles valeurs des indices principaux seront :

$$
n_{x}^{\prime}=\frac{n_{x}}{\sqrt{1+n_{x}^{2} r_{13} E_{3}}} \simeq n_{x}-\frac{1}{2} n_{x}^{3} r_{13} E_{3} ; n_{y}^{\prime} \simeq n_{y}-\frac{1}{2} n_{y}^{3} r_{23} E_{3} ; n_{z}^{\prime} \simeq n_{z}-\frac{1}{2} n_{z}^{3} r_{33} E_{3}
$$

Dans la technique FPTSI on peut montrer qu'à une température $T$ le coefficient électrooptique apparent mesuré est donné par :

$$
r_{i 3}^{\prime}(T)=\frac{2 \Delta I(T)}{E_{3} n_{i}^{2}(T) \Gamma(T) \varphi(T)},
$$

- $\quad n_{i}(T)$ est l'indice de réfraction correspondant à une polarisation de la lumière orientée suivant la direction $i$ avec $i=x, y$ ou $z$;

- $\Delta I(T)$ est la variation d'intensité lumineuse induite par le champ $E_{3}$;

- $\quad \Gamma(T)$ est une fonction de la phase $\varphi(T)$ :

$$
\Gamma(T)=\frac{m \sin \varphi(T)}{2\left(1+m \sin ^{2} \frac{\varphi(T)}{2}\right)^{2}} \text { avec } m=\frac{4 R(T)}{(1-R(T))^{2}}
$$

où $R(T)$ le coefficient de réflexion à la température $T$ et $\varphi(T)=\frac{4 \pi n_{i}(T) e(T)}{\lambda} ; e(T)$ est l'épaisseur de l'échantillon et $\lambda$ la longueur d'onde du faisceau incident.

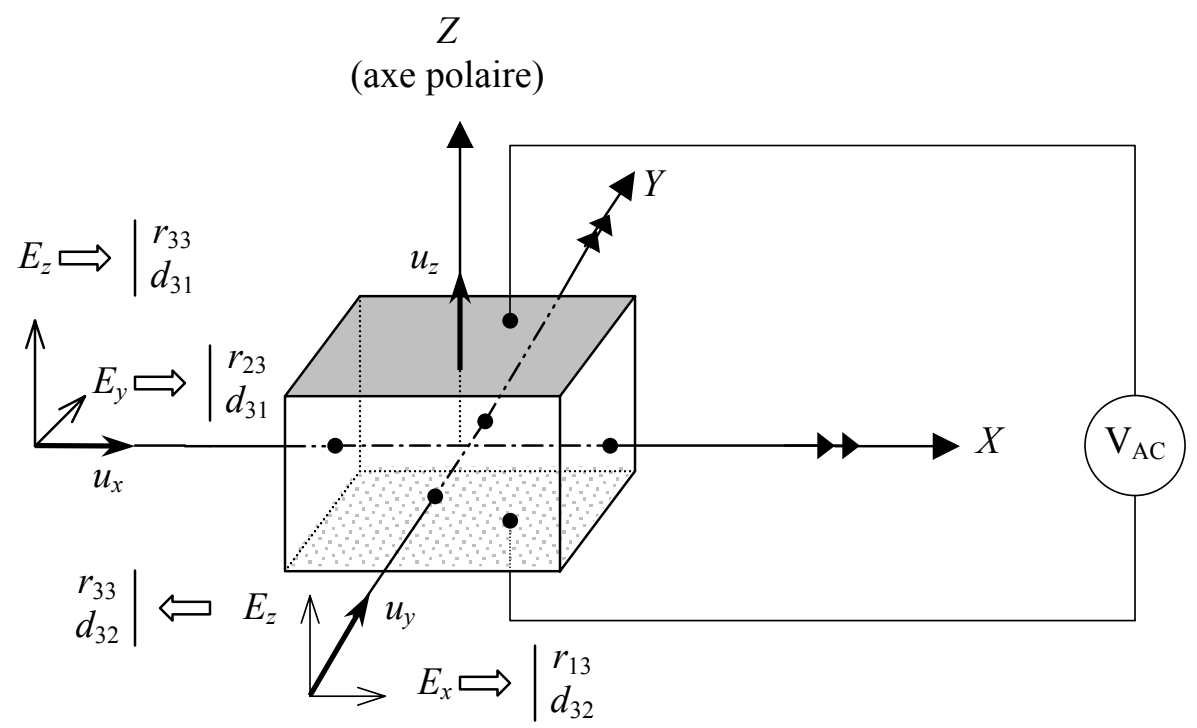

FIG. 17. Configurations géométriques permettant de déterminer les coefficients électro-optiques $r_{i 3}$ dans le cas du système orthorhombique $\mathrm{mm} 2$. Les électrodes sont déposées sur les faces polaires $Z$ de l'échantillon et le faisceau se propage suivant les directions $X$ ou $Y . E_{j}(j=x, y$ ou $z)$ indique la polarisation du rayonnement; $r_{k 3}$ et $d_{3 l}(k=1,2$ ou 3 et $l=1$ ou 2$)$ représentent respectivement les coefficients électro-optiques et piézo-électriques sollicités dans les diverses orientations. $u_{j}(j=x, y$ ou $z)$ sont les déformations engendrées par effet piézoélectrique. 
En première approximation le coefficient électro-optique vrai est obtenu en prenant aussi en compte l'effet piézo-électrique mais en négligeant un effet élasto-optique éventuel. D'après le paragraphe 3.1 précédent, si l'on s'intéresse par exemple à la direction de propagation du faisceau lumineux suivant $\mathrm{X}$, la variation d'épaisseur de l'échantillon est $\mathrm{d}\left(e_{x}\right)=d_{31} E_{3} e_{x}$. La valeur $\mathrm{du}$ coefficient électro-optique $r_{33}$ vrai est alors $r_{33}=r_{33}^{\prime}+\frac{2 d_{31}}{n_{z}{ }^{2}}$. La figure $\underline{17}$ schématise les configurations géométriques permettant la détermination des $r_{i 3}(i=1,2$ ou 3$)$ pour l'exemple cité.

\section{DILATOMÉTRIE INTERFÉROMÉTRIQUE ABSOLUE [10]}

Si les échantillons, que l'on supposera de forme parallélépipédique, sont de dimensions suffisantes, tous les coefficients mentionnés pourront être obtenus simultanément au moyen d'un dilatomètre interférométrique absolu conçu à cet effet. La figure $\underline{18}$ en donne le schéma et la légende, la description.

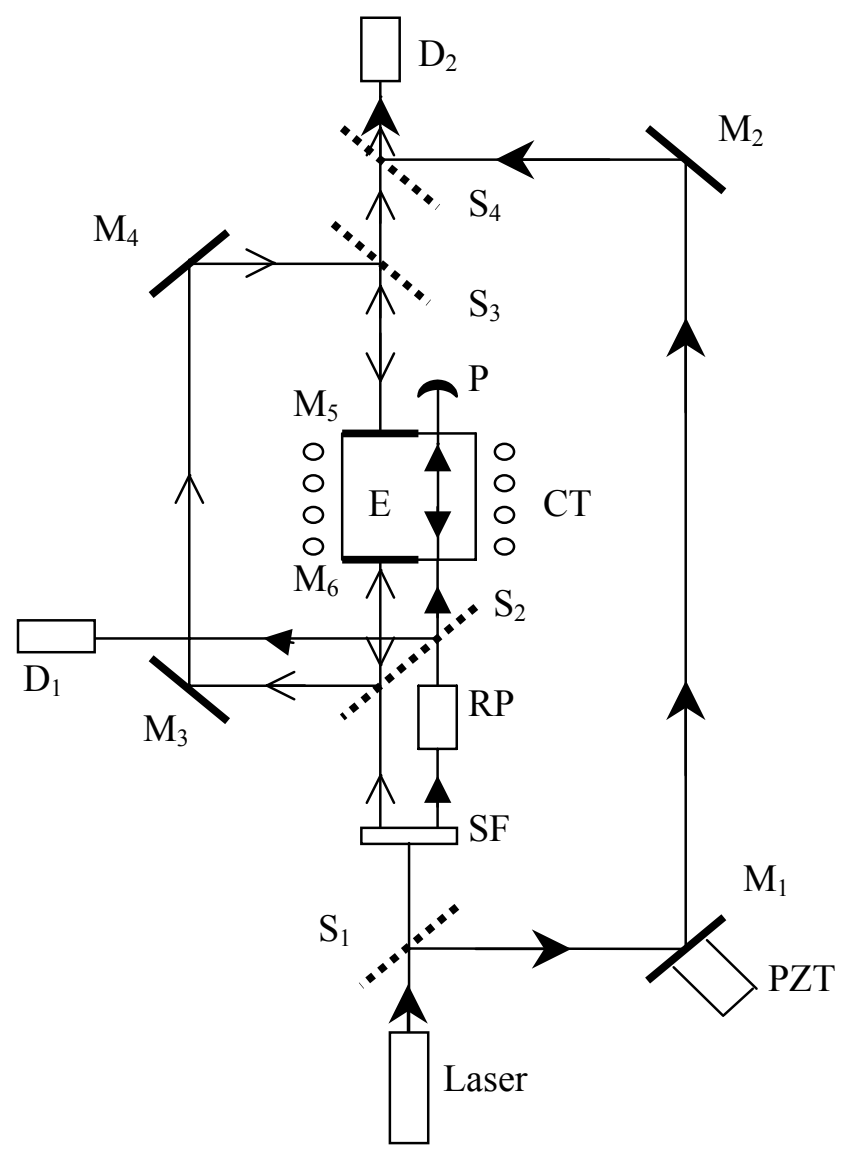

FIG. 18. Dilatomètre interférométrique absolu [10]. Un laser constitue la source de rayonnement. $\mathrm{M}_{1}, \mathrm{M}_{2}, \mathrm{M}_{3}, \mathrm{M}_{4}$ sont des miroirs; $\mathrm{M}_{5}$ et $\mathrm{M}_{6}$ sont les parties métallisées des faces optiques de l'échantillon $\mathrm{E}$, faisant office de réflecteurs. $\mathrm{M}_{1}$ est solidaire d'une cale piézo-électrique PZT permettant un fonctionnement de l'appareil en modulation de phase. $\mathrm{S}_{1}, \mathrm{~S}_{2}, \mathrm{~S}_{3}$ et $\mathrm{S}_{4}$ sont des lames semi-réfléchissantes. $\mathrm{P}$ est un puits de lumière ; SF, RP et CT sont respectivement un séparateur de faisceau, un rotateur de polarisation et un contrôleur de température programmable. La température de l'échantillon est repérée par un thermocouple fin collé sur l'une de ses faces. Le détecteur $\mathrm{D}_{1}$ est employé pour la mesure des coefficients thermo-optiques et électro-optiques (trajet $\longrightarrow$ ) et $\mathrm{D}_{2}$ pour la mesure des coefficients piézo-électriques et d'expansion thermiques (trajet $\longrightarrow$ ). L'ensemble interférométrique est placé sous vide. 
Pour une description détaillée du fonctionnement de l'appareillage et de ses performances on se reportera à la référence [10]. Du point de vue de la précision de mesure, un avantage considérable de cette méthode est de pouvoir déterminer sur un intervalle de température donné l'ensemble des propriétés optiques d'un matériau ainsi que leurs évolutions thermiques, ce que ne permettent pas les méthodes conventionnelles.

\section{BIBLIOGRAPHIE}

[1] U. Willamowski, T. Gross, D. Ristau et Welling, Calorimetric measurement of optical absorption and transmittivity with sub ppm sensitivity, SPIE Proceed. 2775 (1996) 148-158.

[2] J. Mangin, P. Strimer, T. Salva et C. Poignon, Accurate measurement of weak absorption in pyro-electric optical materials, SPIE Proceed. 2775 (1996) 159-165.

[3] F. Bezançon, J. Mangin, P. Strimer et M. Maglione, Accurate determination of the weak optical absorptio of piezo-electric crystals used as capacitive massive bolometers, IEEE J. Quant. Elect. 37 (2001) 1396-2000.

[4] W.P. Mason, Piezo-electric crystals and their application to ultrasonics, D. Van Nostrand Company Inc. Edit. (1950).

[5] J.F. Nye, Physical properties of crystal, Oxford University Press (1976).

[6] A. Yariv et P. Yeh, Optical waves in crystals, John Wiley and Sons (1984).

[7] M. Aillerie, N. Theophanous et M. Fontana, Measurement of the electro-optic coefficients: description and comparison of the experimental techniques, Appl. Phys. B 70 (2000) 317-334.

[8] N Theophanous et N. Arapoyianni, Effect of multiple reflections on retardation-based electrooptic measurements, J. Opt. Soc. Am. A 8 (1991) 1746-1754.

[9] A. Ducharme, L Feinberg, M Ratnaker et G Neurgaonkar, Electro-optic and piezo-electric measurement in photorefractive barium titanate and barium strontium niobate, IEEE J. Quant. Elect. 23 (1987) 2116-2120.

[10] J. Mangin, P. Strimer et L. Lahlou-Kassi, An interferometric dilatometer for the determination of thermo-optic coefficients of NLO materials, Meas. Sci. Technol. 4 (1993) 826-834.

\section{POUR EN SAVOIR PLUS}

D. Royer et E. Dieulesaint, Ondes élastiques dans les solides, tomes I et II, Masson (1996).

M.E. Lines et A.M. Glass, Principles and applications of ferroelectrics and related materials, Oxford University Press (1977).

W.G. Cady, Piezo-electricity: an introduction to the theory and applications of electromechanical phenomena in crystals, Dover Pub. Inc., New-York (1964). 